\title{
A BALLOON-BORNE X-RAY TELESCOPE
}

M. FUJII, M. MATSUOKA, S. MIYAMOTO, J. NISHIMURA

M. ODA, Y. OGAWARA, and S. OHTA

Institute of Space and Aeronautical Science University of Tokyo, Tokyo, Japan

\begin{abstract}
A balloon-borne X-ray instrument with the modulation collimator was designed and constructed to study the structure of solar X-ray flares. The angular resolution was approximately one arc minute. The instrument was used on an occasion of X-ray flare on Sept. 27, 1970.
\end{abstract}

